\title{
Contributions to the Multifunctional Integration for Micromechatronic Systems
}

\author{
M. Grossard Mathieu and M. Chaillet Nicolas \\ CEA, LIST, Service Robotique Interactive, 18 route du Panorama, BP6, FONTENAY \\ AUX ROSES, F- 92265 \\ France \\ FEMTO-ST Institute, Automatic Control and Micro-Mechatronic Systems Department \\ France
}

\section{Introduction}

Mechatronics is the interdisciplinary area related to the integration of mechanical, electronic and control engineering, as well as information technology to design the best solution to a given technological problem. It implies that mechatronics relates to the design of systems, devices and products aimed at achieving an optimal balance between basic mechanics and its overall control. Robotic systems design has certainly been the pioneer field of mechatronic applications.

Due to the increase in the difficulty to miniaturize these advanced (or intelligent) technological products, research in the microrobotic field is required to find novel solutions to design micromechatronic systems. When applying scale reduction to robotic systems usually encountered at the macroscopic scale, the miniaturization step necessarily implies functional integration of these systems. This general trend makes microsystems more and more functionally integrated, which makes them converging towards the adaptronic (or smart structures) concept.

In this coming mechatronic concept, all functional elements of a conventional closed-loop system are existent and at least one element is applied in a multifunctional way. The aim of such a system is to combine the greatest possible number of application-specific function in one single element. It aims at building up a microstructure that is marked by minor complexity and high functional density (Fig. 1).

The key idea followed in the micromechatronic design is that three of the four components (i.e. sensors, actuators and mechanical structure) in smart microrobotic structures are made of a single functional (or active) material, such as piezoelectric or shape memory alloys materials. They can perform actuation or/and sensing functions by interchanging energy forms (for example, electric energy, magnetic energy and mechanical energy). 


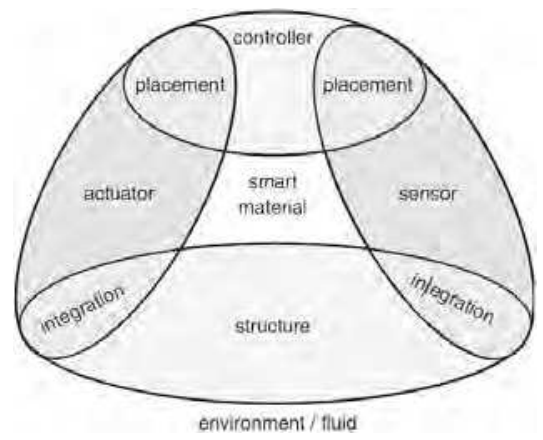

Fig. 1. Integrated smart structure (Hurlebaus, 2005).

Most often, these integrated microdevices are compliant mechanisms, i.e. single-bodies, elastic continua flexible structures that transmit a motion by undergoing elastic deformation, as opposed to jointed rigid body motions of conventional articulated mechanisms. Using compliant mechanisms for the design of small scale systems is of a great interest, because of simplified manufacturing, reduced assembly costs, reduced kinematic noise, no wear, no backlash, and ability to accommodate unconventional actuation schemes when they integrate active materials.

These micromechatronic devices consist of a dynamic system combining a flexible mechanical structure with integrated multifunctional materials. For the simulation and optimization of such microsystems, control and system theory together with proper modeling of the plant are to be applied. The finite element method is a widespread tool for numerical simulation and structural modeling that can include multiphysics due to the cross coupling effects of the active material. Afterwards, the efficiency and proper positioning of actuators and sensors in these systems can be analyzed using the concepts of controllability and observability. Then, the state-space representation is desirable to achieve model reduction and to perform control design methodologies.

A general overview of design specificities including mechanical and control considerations for micromechatronic structure is firstly presented in this chapter. Performance criteria including mechanical performances, spillover treatment, model reduction techniques and robust control are briefly presented afterwards.

Finally, an example of a new optimal synthesis method to design topology and associated robust control methodologies for monolithic compliant microstructures is presented. The method is based on the optimal arrangement of flexible building blocks thanks to a multicriteria genetic algorithm. It exploits the piezoelectric effect, thus making realistic the adaptronic concept, i.e. integration of the actuation/sensing principle inside the mechanical structure.

\section{Design and control specificities of active flexible micromechatronic systems}

In the section, a particular attention is drawn on the approach used for modelling and optimizing these micromechanisms design. 


\subsection{Design and modelling}

When compared to macroscale mechatronic systems, design of micromechatronic systems needs some particular attention. Indeed, this miniaturization step implies to rethink the main functionalities of the traditional systems in accordance to the specificities of the microscale:

- their microstrucure, as well as their fabrication and microassembly process ;

in many applications including Micro Electro Mechanical Systems (MEMS) (Lee 2003), (Chang 2006), (Kota 1994), surgical tools (Frecker 2005) (Houston 2007), etc, compliant mechanisms have already been used. They are single-body, elastic continua flexible structures, that deliver the desired motion by undergoing elastic deformation, as opposed to jointed rigid body motions of conventional mechanisms. There are many advantages of compliant mechanisms, among them: simplified manufacturing, reduced assembly costs, reduced kinematic noise, no wear, no backlash, high precision, and ability to accommodate unconventional actuation schemes.

- their actuators and sensors with high resolution and small size ;

new ways for producing actuation and sensing need to be studied in their physical principle, as well as their good adaptability for the achieving tasks at the microscale in term of displacement, force, controllability, observability, etc. The use of active functional material (also called multifunctional materials), which can convert energy from one form to the other, are thus widespread in the context of micro-actuator/sensor design.

- their control methodology and implementation.

The design of controllers for active flexible micromechanisms is a challenging problem because of nonlinearities in the structural system and actuators/sensors behavior, nonavailability of accurate mathematical models, a large number of resonant modes to accurately identify and control. Thus, robust control design methods need to be used.

Most often, modelling and simulating active flexible mechanisms can be made following several steps sketched on Fig. 2. Starting from the chosen active material (such as piezoelectric ceramics or magnetostrictive materials), coupled with some specific boundary conditions and system geometry inherent to the problem, the global equations for the system behavior are established using the equations of dynamic equilibrium and kinematics. Then, the finite element (FE) method is generally used for discretizing the spatial distribution of displacements within the flexible structure: it reduces the problem formulation to a discrete set of differential equations. In this manner, multiphysics problem can be treated when considering the electromechanical (in the case of piezoelectric materials) or magnetomechanical (in the case of magnetostrictive materials) couplings of the active materials. In the perspective of controlling these mechanisms, this dynamic input/output model is expressed using state-space formalism. Structural models obtained by using FE method exhibit a huge number of degrees of freedom. Thus, the resulting full order model has to be drastically reduced thanks to reduction techniques. Usual techniques of reduction consist in selecting the most influent modes that lie in the frequency spectrum of interest, i.e. those that are strongly controllable and observable with the actuator/sensor configuration.

Some examples of software tools related to the simulation (and, in some restrictive case, the optimization process as well) of smart structures can be found in (Janocha 2007). 


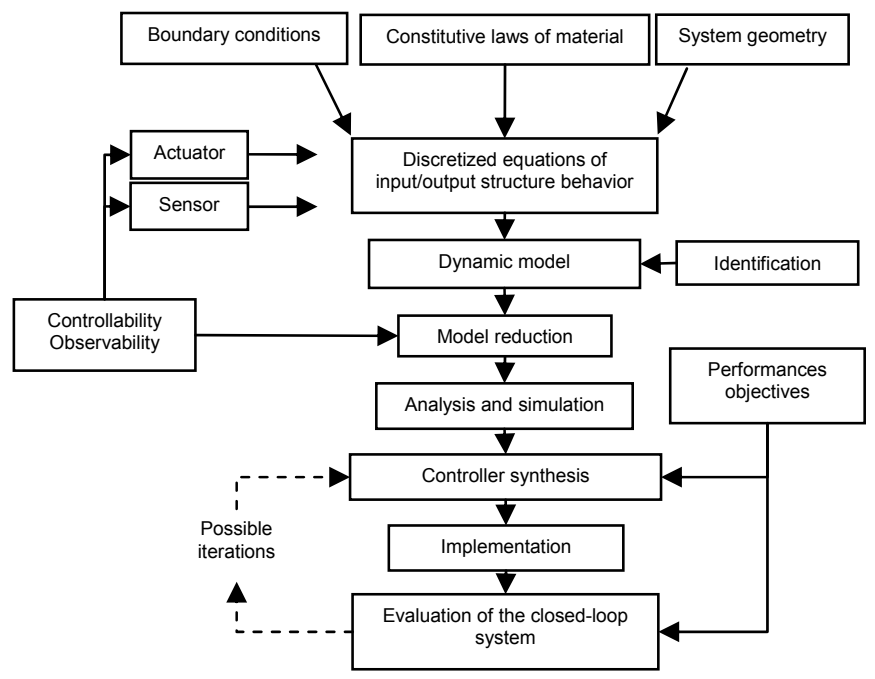

Fig. 2. General approach for modelling and testing active flexible micromechanisms

\subsection{Design optimization}

Modeling, simulating and controlling integrated flexible structures imply a parameterization of the considered system (geometry, material, etc). In link with the application task, parametric studies are generally led to determine the most adequate design for the structure, the actuators/sensors, the controller, etc. Thus, this design process can be formalized under an optimization problem to select the optimal solution(s).

A general strategy needs to be appropriate to deal with the coupling problem between the structure, the actuators and sensors, and the control of the system.

Generally, a decomposition approach is privileged, especially for complex problem. The optimization of some parts of the system is separately considered under several constraint hypothesis. For example, some papers deal solely with control systems for a specified structure. Other works deals with optimal actuator placement on a predetermined flexible structure, or with coupling flexible structures for single actuators, etc. A current work concerning design methodologies and application of formal optimization methods to the design of smart structures and actuators can be found in (Frecker 2000).

In the following, a particular attention is made on the use of piezoelectric ceramic as an active material for microrobotic tools. Indeed, one type of smart material-based actuator typically used to actuate compliant structures is piezoelectric ceramic PZT actuators: when compared to other conventional actuation principles at small scales, they have very appealing properties in the sense of micromechatronic design. When integrated inside a compliant mechanism, piezoelectric actuators can exert actuation forces to the host structure without any external support. They can also be manufactured into the desired shape, while making realistic the realization of piezoelectric monolithic compliant mechanisms, such as microgrippers (Breguet 1997). Piezoelectric actuation is mostly used for microrobot design in order to achieve nanometric resolutions, and has naturally become widespread in micromanipulation systems (Agnus 2005). 
However, one limitation of piezoelectric actuators is that they are capable of producing only about $0.1 \%$ strain, resulting in a restricted range of motion. A number of papers only address the problem of optimally designing coupling structures to act as stroke amplifiers of the piezoelectric actuator (Kota 1999), (Lau 2000). Opposite to these methods, where the piezoelectric elements in the structure are predetermined, a large body of work related to optimization of active structures deals with the optimal location of actuators on a given structure (Barboni 2000). Another general approach to optimally design actuated structures is to simultaneously (Maddisetty 2002) or separately (Abdalla 2005) optimize the actuator size. Finally, few studies consider the topology optimization (shape) of monolithic PZT active structures (Nelli Silva 1999).

\subsection{Dynamics of the flexible micromechanisms}

There are a number of difficulties associated with the control of flexible structures (amongst them, variable resonance frequencies, highly resonant dynamics and time-varying states subjected to external disturbances).

For example, since the dynamic model of a flexible structure is characterized by a large number of resonant modes, accurate identification of all the dominant system dynamics often leads to very high order model. Thus, a model reduction is required by the designer. A number of approaches for model reduction have been developed, such as model reduction via balanced realization (Moore 1981). But, this reduction model step is quite delicate because of spillover effect, that is to say when unwanted interactions between the controlled system and neglected structural modes lead to instability.

Thus, an important condition for a controlled dynamic system is to guarantee its stability. Moreover, the stability of such controlled dynamic system has to be robust, that is to say it must stabilizes the real system in spite of modelling errors or parameters changes. Thus, traditional robust control system design techniques such as LQG, $\mathrm{H}_{2}$ and $\mathrm{H}_{\infty}$ commonly appear in research works (Abreu 2003), (Halim 2002a), (Halim 2002b). The performances of such high authority controllers have to take into account model uncertainties and modelling errors introduced by model truncation.

For some specific class of flexible structures, which can be modelled as collocated resonant systems, active damping dissipative controllers (for example, Positive Position Feedback, Integral Force Feedback, Direct Velocity Feedback...) have proven to offer great robustness, performance, and ease of implementation relatively to traditional techniques. On the contrary of the advanced techniques, the direct use of dissipative collocated controllers can have the advantages to produce control systems of low order and good robustness, associated with high dynamic performance. These techniques are often focused on damping the dominant modes (Aphale 2007). The natural modes of the system must be controlled using proper actuators and sensors positions ('Control authorithy'): actuator and sensor positions are sought for influencing (controllability) and sensing (observability) the modal oscillations.

\section{Example of an optimal synthesis tool for designing smart microrobotic structure}

In this paragraph, a method developed for the optimal design of piezoactive compliant micromechanisms is presented. It is based on a flexible building block method, which uses 
an evolutionary approach, to optimize a truss-like planar structure made of passive and active building blocks, made of piezoelectric material. An electromechanical approach, based on a mixed finite element formulation, is used to establish the model of the active piezoelectric blocks. From the first design step, in addition to conventional mechanical criteria, innovative control-based metrics can be considered in the optimization procedure to fit the open-loop frequency response of the synthesized mechanisms. In particular, these criteria have been drawn here to optimize modal controllability and observability of the system, which is particularly interesting when considering control of flexible structures.

More specific details on this method can be found in (Bernardoni 2004a), (Bernardoni 2004b), (Grossard 2007a), (Grossard 2007b).

\subsection{Compliant building blocks}

Two libraries of compliant elements in limited number are proposed in our method. These bases are composed respectively of 36 and 19 elements of passive and piezoactive blocks, made of beams assembly (Fig. 3). They are sufficient to build a high variety of topologies. In particular, the various topologies of piezoelectric active blocks allow them to furnish multiple coupled degrees of freedom, thus generating more complex movements with only one building block.

\subsection{Principles of the method and design parameters}

The specification of a planar compliant mechanism problem considers specific boundary conditions: fixed frame location, input (actuators), contacts and output (end-effector). In particular, a particular attention is drawn on the integrated piezoactive elements taken from the active library as actuator. The design method consists in searching for an optimal distribution of allowed building blocks, as well as for the optimal set of structural parameters and materials. The location of fixed nodes and that of the piezoactive blocks can also be considered as optimisation parameters. The topology optimization method uses a genetic algorithm approach, which allows true multicriteria optimization and the use of these discrete variables (Fig. 4). The algorithm is structured as follows: discrete variable parameterization of compliant mechanisms considering conception requirements (mesh size, topology, material and thickness, boundary conditions), evaluation of individuals (design criteria calculation), and stochastic operators for the optimization (modification of compliant mechanisms description). 


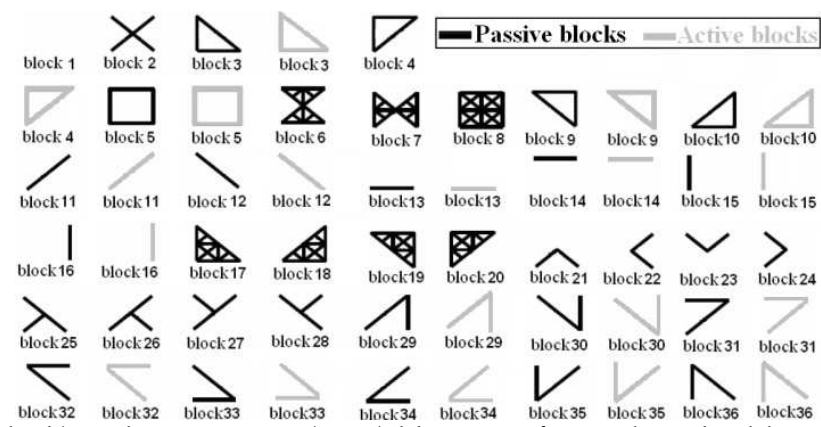

Fig. 3. Passive (black) and piezoactive (grey) libraries of compliant building blocks, for planar compliant mechanisms synthesis.

Many fitness functions are available in our method, thus allowing the optimal design of devices within a wide schedule of conditions: static mechanical fitness (free displacement and blocking force at the output port, geometric advantage, mechanical advantage, etc.), various dynamic control-oriented metrics have been newly implemented to meet specific control requirements for microrobotics devices. Obviously, the design strategy depends on the metrics chosen, which must be based on the real needs for the device use.

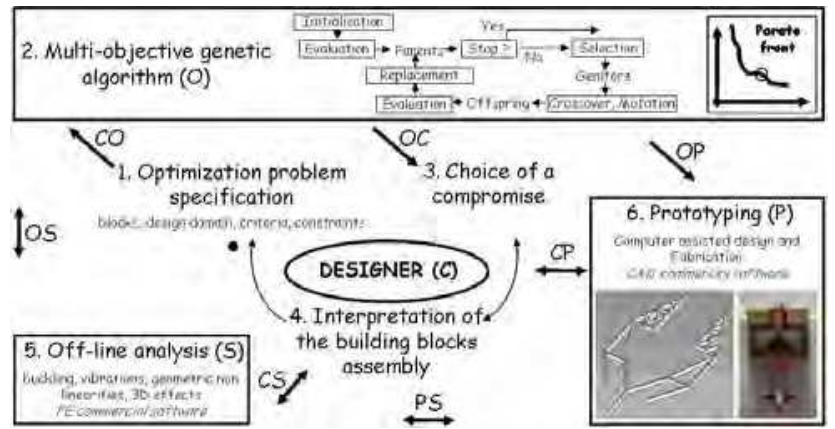

Fig. 4. Flowchart of the optimal design method of compliant structures (multicriteria optimization).

\subsection{Electromechanical FE model of the piezoelectric structure}

In our method, it is assumed that the compliant mechanisms are undergoing structural deformations, mainly due to the bending of the beams constituting the blocks. Thus, the models of the blocks are obtained considering Navier-Bernoulli beam type finite elements. Structural parameters of each rectangular block are height, width and thickness. Material characteristics of each block are parameterized by Young's modulus, Poisson's ratio, yield strength, density, and piezoelectric coefficients for the piezoactive blocks.

The piezoceramic beams constituting the active blocks are perfectly bonded to electrodes at their lower and upper faces (Fig. 5). Exploiting the transverse effect of piezoelectricity, longitudinal deformation $S_{11}$ along $\mathrm{L}$ dimension is generated under the transverse electric field $E_{3}$. Considering the one-dimensional form of piezoelectricity equation along the length direction of the beam, the piezoelectric coupling matrix $\mathbf{d}$ and the stress-free electric 
permittivity matrix $\varepsilon$ are each represented by a single coefficient, $d_{31}$ and $\varepsilon_{33}$ respectively, and the electric-free compliance matrix $\mathbf{s}$ is represented by $s_{11}$. Hence, within the piezoelectric beam, the constitutive relations for the strain $S_{11}$ and electric displacement $D_{3}$, as functions stress $\mathrm{T}_{11}$ and electric field $\mathrm{E}_{3}$, take the form:

$$
\left(\begin{array}{l}
\mathrm{S}_{11} \\
\mathrm{D}_{3}
\end{array}\right)=\left[\begin{array}{ll}
\mathrm{s}_{11} & \mathrm{~d}_{31} \\
\mathrm{~d}_{31} & \varepsilon_{33}
\end{array}\right]\left(\begin{array}{l}
\mathrm{T}_{11} \\
\mathrm{E}_{3}
\end{array}\right)
$$

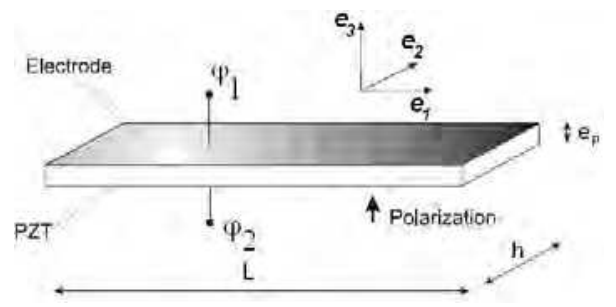

Fig. 5. Thickness-polarized piezoelectric beam transducer with electroded surfaces, and orientation in the material reference frame $\left(\mathrm{e}_{1}, \mathrm{e}_{2}, \mathrm{e}_{3}\right) . \varphi_{1}$ and $\varphi_{2}$ denotes the electric potential of the electrodes.

From Hamilton's principle modified for general electromechanical system, the model of the active beam takes the following form:

$$
\mathrm{M}_{\mathrm{b}} \ddot{\eta}_{\mathrm{b}}+\mathrm{K}_{\mathrm{b}} \eta_{\mathrm{b}}=\mathrm{G}_{\mathrm{b}} \Phi_{\mathrm{b}}+\mathrm{Fr}_{\mathrm{b}}
$$

where $\mathbf{M}_{\mathrm{b}}, \mathbf{K}_{\mathrm{b}}$ and $\mathbf{G}_{\mathrm{b}}$ are respectively the mass, stiffness and electromechanical coupling beam matrices. $\boldsymbol{\Phi}_{\mathrm{b}}=\left[\begin{array}{ll}\varphi_{1} & \varphi_{2}\end{array}\right]^{\mathrm{t}}$ is the vector representing the electric potentials on the upper and lower faces of the piezoelectric beam. Matrix $\mathbf{G}_{b}$ induces piezoelectric loads, which makes the actuator beam expand (or contract) proportionally to the external controlled potential difference $\left(\varphi_{1}-\varphi_{2}\right)$. The forces vector $\mathbf{F r}_{\mathrm{b}}$ is due to the variational mechanical work terms. Displacement field is related to the corresponding node values $\boldsymbol{\eta}_{\mathrm{b}}$ by the mean of the shape functions, calculated under Euler-Bernoulli beam assumptions. Detailed derivations can be readily found in finite element textbooks, and corresponding matrices in (Grossard 2007).

The stiffness, damping, and mass matrices of each block are then calculated numerically, considering every combination of the discrete values allowed for the structural optimization variables. Then, the global dynamic behaviour of a structure results from the mass, damping, stiffness and electromechanical coupling matrices assembly of the constitutive blocks, and is done at each step for each individual during the optimization process.

The conservative dynamic behaviour of a structure is described through its mass $\mathbf{M}_{\mathrm{g}}$, stiffness $\mathbf{K}_{\mathrm{g}}$ and electromechanical coupling $\mathbf{G}_{\mathrm{g}}$ matrices, obtained by the assembly in of the matrices of the blocks constituting the structure. 


\subsection{State-space model for flexible structure}

Each flexible structure synthesized by our method is defined as a finite-dimension, controllable and observable linear system with small damping and complex conjugate poles (Lim 1993). Its undamped dynamic behavior is modeled by the following second-order differential matrix equations:

$$
\begin{gathered}
\mathrm{M}_{\mathrm{g}} \ddot{\eta}_{\mathrm{g}}+\mathrm{K}_{\mathrm{g}} \eta_{\mathrm{g}}=\mathrm{E}_{\mathrm{g}} \mathrm{u} \\
\mathrm{y}=\mathrm{F}_{\mathrm{g}} \eta_{\mathrm{g}}
\end{gathered}
$$

$\eta_{\mathrm{g}}$ is the nodal displacement vector, $\mathrm{u}$ is the input vector which defines the controlled command of the actuator. For example, in case of a piezoelectric actuation scheme, $u$ is defined by $\Phi_{\mathrm{g}}$. $\mathrm{y}$ is the output vector, defined from the output displacement matrix $\mathrm{F}_{\mathrm{g}}$. Each element of $\mathrm{u}$ (resp. y) denotes a physical actuator (resp. sensor) whose related degree of freedom is defined by the location of the nonzero entry in the corresponding column in $E_{g}$ (resp. row in $\mathrm{F}_{\mathrm{g}}$ ).

By means of modal decomposition, a solution of the form

$$
\eta_{\mathrm{g}}(\mathrm{t})=\sum_{\mathrm{i}} \psi_{\mathrm{i}} \mathrm{q}(\mathrm{t})=\psi \mathrm{q}(\mathrm{t})
$$

is considered, which consists of a linear combination of mode shapes $\psi_{\mathrm{i}}$. The eigenvectors matrix $\psi$ and corresponding eigenfrequencies $\omega_{\mathrm{i}}$ are obtained as solutions of the vibration eigenproblem (Grossard 2007). Replacing $\eta_{\mathrm{g}}$ by $\psi \mathrm{q}$ in (Eq. 3), multiplying on the left by $\psi^{\mathrm{t}}$, the induced orthogonality relationships in modal form lead to:

$$
\begin{gathered}
\ddot{\mathrm{q}}+\operatorname{diag}\left(2 \xi_{\mathrm{i}} \omega_{\mathrm{i}}\right) \dot{\mathrm{q}}+\operatorname{diag}\left(\omega_{\mathrm{i}}^{2}\right) \mathrm{q}=\psi^{\mathrm{t}} \mathrm{E}_{\mathrm{g}} \mathrm{u} \\
\mathrm{y}=\mathrm{F}_{\mathrm{g}} \psi \mathrm{q}
\end{gathered}
$$

In this equation, diagonal damping by using Basil's hypothesis has been introduced. Thus, $\xi_{\mathrm{i}}$ is here the $\mathrm{i}$-th modal damping ratio. This hypothesis can be made because the system to control is slightly damped in the low-frequency band, where the modes are well separated. One interesting state vector $x$ consists of modal velocities and frequency weighted modal displacements:

$$
\mathrm{x}=\left(\begin{array}{ccccc}
\dot{\mathrm{q}}_{1} & \omega_{1} \mathrm{q}_{1} & \ldots & \dot{\mathrm{q}}_{\mathrm{n}} & \omega_{\mathrm{n}} \mathrm{q}_{\mathrm{n}}
\end{array}\right)^{\mathrm{t}}
$$

with the advantage that the elements of state vector corresponding to each mode are about the same magnitude. This yields the matrices triplet $(\mathrm{A}, \mathrm{B}, \mathrm{C})$ which denotes the modal state-space representation of a structure as stated below, 


$$
\begin{gathered}
\dot{\mathrm{X}}=\mathrm{AX}+\mathrm{BU} \\
\mathrm{Y}=\mathrm{CX}
\end{gathered}
$$

The matrices take the forms $\mathrm{A}=\operatorname{diag}\left(\begin{array}{lll}\mathrm{A}_{1} & \ldots & \mathrm{A}_{\mathrm{n}}\end{array}\right), \quad \mathrm{B}=\left(\begin{array}{lll}\mathrm{B}_{1}{ }^{\mathrm{t}} & \ldots & \mathrm{B}_{\mathrm{n}}{ }^{\mathrm{t}}\end{array}\right)^{\mathrm{t}}$ and $\mathrm{C}=\left(\begin{array}{lll}\mathrm{C}_{1} & \ldots & \mathrm{C}_{\mathrm{n}}\end{array}\right)$, with :

$$
\mathrm{A}_{\mathrm{i}}=\left[\begin{array}{cc}
-2 \xi_{\mathrm{i}} \omega_{\mathrm{i}} & -\omega_{\mathrm{i}} \\
\omega_{\mathrm{i}} & 0
\end{array}\right], \mathrm{B}_{\mathrm{i}}=\left[\begin{array}{c}
\psi_{\mathrm{i}}^{\mathrm{t}} \mathrm{E}_{\mathrm{g}} \\
0
\end{array}\right], \mathrm{C}_{\mathrm{i}}=\left[\begin{array}{ll}
0 & \frac{1}{\omega_{\mathrm{i}}} \mathrm{F}_{\mathrm{g}} \Psi_{\mathrm{i}}
\end{array}\right]
$$

Let us note that A matrix depends on the structure itself (eigenfrequencies and modal damping ratios), $\mathrm{B}$ matrix on the location and class of actuators, and $\mathrm{C}$ matrix on location and class of sensors. This modal state is considered to be a physical coordinate because of its direct physical link to structural mode shapes.

\subsection{Useful measures for fitting the frequency response of flexible systems}

From the computation of the linear state model of compliant systems, an optimal topology design strategy is derived taking into account control considerations. In the method, numerical criteria help reaching input-output open-loop system performances with specific operation requirements.

Since the dynamic model of a flexible structure is characterized by a large number of resonant modes, accurate identification of all the dominant system dynamics often leads to very high order model. Thus, a model reduction is required.

A first criterion has been drawn to optimize the reduced-model accuracy of the systems, while limiting spillover effects (Fig. 6). Given a set of structures to optimize, the optimal structures are chosen as the ones guaranteeing the highest joint controllability and observability for all the modes in the bandwidth of interest (i.e. resonance peaks amplitudes must be maximized in the frequencies bandwidth $\left[0 ; \omega_{c}\right]$ to increase authority control on these dominant modes), while providing the minimum joint controllability and observability of the neglected modes (i.e. the amplitudes of resonance peaks after cut-off frequency must be minimized to increase gain margin and to limit modes destabilization in this area). This criterion will enable the rise of structures with accurate reduced model, based on a few highly dominant modes, allowing the easy identification and computation of state model, well adapted to further design and implementation of the control system.

To improve simultaneously the control authority on the $\mathrm{k}$ first dominant modes and the accuracy of the reduced order model, the first new criteria implemented in the method is the following:

$$
J_{1}^{k}=\frac{\sum_{i=1}^{k} \sigma_{i}}{\sum_{i=k+1}^{n} \sigma_{i}}
$$


where $\sigma_{i}$ are the Hankel Singular Values (HSV) defined in their modal form for flexible structures (Lim 1996):

$$
\sigma_{i}=\frac{\sqrt{\left(\psi_{i} \mathrm{~F}_{\mathrm{g}}\right)^{\mathrm{t}}\left(\psi_{\mathrm{i}} \mathrm{F}_{\mathrm{g}}\right)\left(\psi_{\mathrm{i}}^{\mathrm{t}} \mathrm{E}_{\mathrm{g}}\right)\left(\psi_{\mathrm{i}}^{\mathrm{t}} \mathrm{E}_{\mathrm{g}}\right)^{\mathrm{t}}}}{4 \xi_{\mathrm{i}} \omega_{\mathrm{i}}^{2}}
$$

The corresponding i-th HSV is proportionally linked to the maximum amplitude value of the frequency response at $\omega_{i}$ natural pulsation. The $k$ first resonant modes (where $k<n$ ) will be optimized to guarantee high HSV compared to the ones out of the bandwidth. The modal states with small HSV are both weakly controllable and weakly observable, and will be removed from the reduced-system.

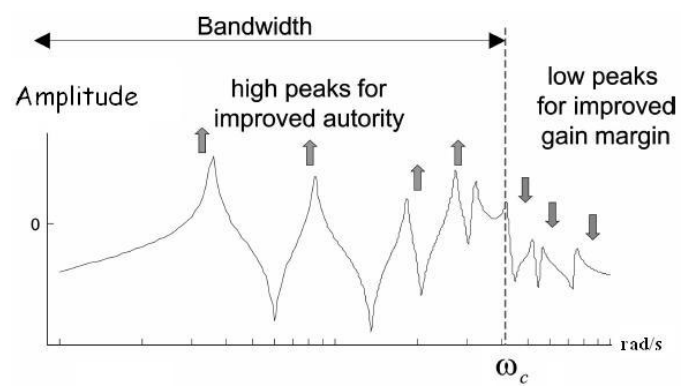

Fig. 6. Desired form of the open-loop frequency response function.

A second criteria relating to control of flexible is particularly interesting. One major characteristic of a collocated system is the interlacing of poles and zeros along the imaginary axis. For a lightly damped structure, poles and zeros are located in the left half-part in the pole-zero map. Such systems are minimum of phase, so that collocated systems are known to possess interesting properties. Vibration control of flexible structures involving collocated characteristics was discussed in (Martin 1978). Control was shown to have simple stability criteria due to the alternating poles and zeros pattern.

An evaluation function was implemented in our method to be used in the optimization process in order to obtain systems designs with collocated type open-loop transfer function, forcing the resonances (poles) and antiresonances (zeros) alternating in the reduced model. Inspired by (Martin 1976), it can be shown that the maximization of the following discrete criterion will imply the interlacing pole-zero pattern exhibited by a collocated transfer function:

$$
\mathrm{J}_{2}^{\mathrm{k}}=\left|\sum_{\mathrm{i}=1}^{\mathrm{k}} \operatorname{sign}\left(\left(\mathrm{F}_{\mathrm{g}} \psi_{\mathrm{i}}\right)\left(\psi_{\mathrm{i}}^{\mathrm{t}} \mathrm{E}_{\mathrm{g}}\right)\right)\right|
$$

where $\operatorname{sign}()=.\{-1 ; 0 ;+1\}$ according to the argument sign. The sum over $\mathrm{i}$ concerns all the modes contained in the frequency spectrum of the first $\mathrm{k}$ dominant modes, where the alternative is desired. 


\subsection{Example of an optimal synthesis of an integrated flexible piezoelectric actuator}

The concepts presented previously have been applied to the design of a microgripper actuator, considering a multi-criteria optimization problem, with both static mechanical (free stroke and blocking force at the output node of the structure) and control-oriented $\mathrm{J}_{1}$ and $\mathrm{J}_{2}$ fitnesses.

The synthesis of a symmetric monolithic microactuation mechanism, made of a single piezoelectric material (PIC151 from PI Piezo Ceramic Technology) has been made using our method. From the set of structures results, one pseudo-optimal solution, whose topology is presented on Fig. 7, is chosen to illustrate performances.
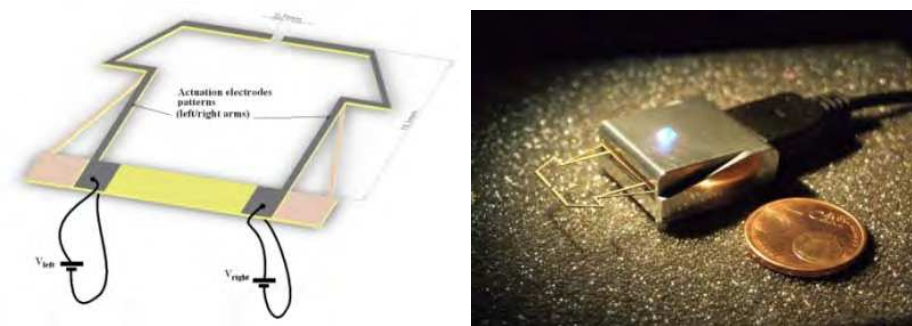

Fig. 7. On the left, model of the piezoeletric device with top face electrode patterns $-V_{\text {left }}$ (resp. $V_{\text {right }}$ ) is the controlled input for actuating the left (resp. right) arm. On the right, photo of the prototyped piezoelectric device, obtained by laser cutting.

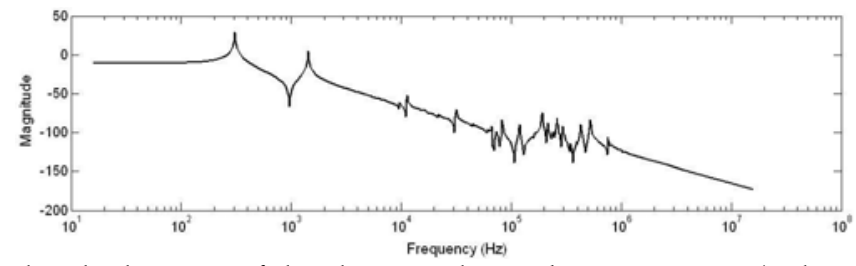

Fig. 8. Bode amplitude diagram of the chosen solution between input (voltage $u$, in $V$ ) and arm output (deflection in $\mu \mathrm{m}$ ) simulated by our method.

Each arm of such a microgripper is able to produce $\pm 10.69 \mu$ m movement range when $\pm 100 \mathrm{~V}$ is applied on the actuation electrodes. A blocking force of about $840 \mathrm{mN}$ is also produced. Moreover, this solution is an example of structure with interesting control-oriented criteria (Fig. 8): the authority control on the two first resonant modes is well optimized, resulting in an important roll-off after the second resonance. As expected, this structure exhibits an alternating pole/zero pattern in the spectrum of interest.

\section{Conclusion}

A brief overview of design specificities and strategies including mechanical and control considerations for micromechatronic structures has been presented. Designing, modelling and controlling flexible microscale structures actuated by active materials are a quite complex task, partly because the designer has to deal with several problems. Amongst them, 
specific mechanical performances, spillover treatment, model reduction techniques and robust control have been highlighted in this chapter.

To help the design of such systems, an example of a systematic optimal design method for smart compliant mechanisms has been particularly presented here. This method can consider a smart compliant mechanism as an assembly of passive and active compliant building blocks made of PZT, so that actuators are really integrated in the structure. Complex multi-objective design problems can be solved, taking advantage of versatile criteria to synthesize high performance microrobotic flexible mechanisms designs. In addition to classical mechanical criteria, currently encountered in topology optimization (i.e. force and displacement maximization), our method considers now simultaneously efficient control-based criteria.

Open-loop transfer considerations lead to two new efficient numerical criteria. A first criterion can modulate resonances amplitudes of its frequency response function in a spectrum of interest. A second criterion can force minimum-phase system property. These two criteria, coupled with mechanical ones, help designing non-intuitive compliant mechanisms. This optimization strategy was tested for the optimal design of a microgripper actuator. The results obtained have proved that the method can furnish innovative and efficient solutions.

\section{References}

Abdalla M., and al. (2005), Design of a piezoelectric actuator and compliant mechanism combination for maximum energy efficiency, Smart Material and Structures, vol. 14, pp. 1421-1430, 2005

Abreu G. L. C., Ribeiro M., Steffen J. F. (2003), Experiments on optimal vibration control of a flexible beam containing piezoelectric sensors and actuators, Journal of Shock and Vibration, vol. 10, pp. 283-300, 2003

Agnus J., Nectoux P. , Chaillet N. (2005), Overview of microgrippers and micromanipulation station based on a MMOC microgripper, Proc. of the IEEE International Symposium on Computational Intelligence in Robotics and Automation, pp. 117-123, 2005

Aphale S. S., Fleming A.J., Moheimani S. O. R. (2007), Integral resonant control of collocated smart structures, Smart Materials and Structures, vol.16, pp. 439-446, 2007

Barboni R., and al. (2000), Optimal placement of PZT actuators for the control of beam dynamics, Smart Material and Structures, pp. 110-120, 2000

Bernardoni P., and al. (2004a), A new compliant mechanism design methodology based on flexible building blocks, Smart Material and Structures, vol. 5383, pp. 244-254, USA, 2004

Bernardoni P. (2004b), Outils et méthodes de conception de structures mécaniques à déformations réparties et actionnement discret - applications en microrobotique, $P h D$ Thesis realized at the CEA, University Paris 6, France, 2004

Breguet J.M. (1997), and al., Monolithic piezoceramic flexible structure for micromanipulation, 9th International Precision Engineering Seminar and 4th International Conference on Ultraprecision in Manufacturing Engineering, pp. 397-400, Braunschweig Germany, 1997 
Chang H.C., Tsai J.M.L., Tsai H.C., Fang W. (2006), Design, fabrication, and testing of a 3DOF HARM micromanipulator on (111) silicon substrate, Sensors and Actuators, vol. 125, pp. 438-445, 2006

Frecker M., Canfield S. (2000), Optimal design and experimental validation of compliant mechanical amplifiers for piezoceramic stack actuators, Journal of Intelligent Material Systems and Structures, vol. 11, pp. 360-369, 2000

Frecker M., Haluck R. (2005), Design of a multifunctional compliant instrument for minimally invasive surgery, Journal of Biomedical Engineering, vol. 127, pp. 990-993, November 2005

Grossard M., Rotinat-Libersa C., Chaillet N. (2007a), Redesign of the MMOC microgripper piezoactuator using a new topological method, IEEE/ASME International Conference on Advanced Intelligent Mechatronics, Zürich, Switzerland, 2007

Grossard M., Rotinat-Libersa C., Chaillet N., Perrot Y. (2007b), Flexible building blocks method for the optimal design of compliant mechanisms using piezoelectric material, 12th IFToMMWorld Congress, Besançon, France, 2007

Halim D., Moheimani S. O. R. (2002a), Experimental implementation of spatial H1 control on a piezoelectric laminate beam, IEEE/ASME Transactions on Mechatronics, vol. 4, pp. 346-356, 2002

Halim D., Moheimani S. O. R. (2002b), Spatial H2 control of a piezoelectric laminate beam: experimental implementation, IEEE Transactions on Control System Technology, vol. 10, pp. 533-546, 2002

Houston K., Sieber A., Eder C., Tonet O., Menciassi A., Dario P. (2007), Novel Haptic Tool and Input Device for Real Time Bilateral Biomanipulation addressing Endoscopic Surgery , Proc. of the 29th Annual International Conference of the IEEE EMBS, Lyon, France, August 23-26, pp. 198-201, 2007

Hurlebaus S. (2005). Smart Structures - Fundamentals and Applications, Lecture Notes, Texas A\&M University, Zachry Department of Civil Engineering

Janocha, H. (2007). Adaptronics and smart structures - Basics, Materials, design, and Applications, Springer Editor, ISBN 978-3-540-71965-6, Berlin Heildeberg New-York

Kota S., Ananthasuresh G.K., Crary S.B., and Wise K. D. (1994), Design and fabrication of micro-electromechanical systems, ASME Journal of Mechanical Design, vol. 116, pp. 1081-1088, 1994

Kota S. (1999), "Tailoring unconventional actuators using compliant transmissions: design methods and applications", IEEE/ASME Transactions on Mechatronics, vol. 4, pp. 396408, December 1999

Lau G. K., and al. (2000), Systematic design of displacement - amplifying mechanisms for piezoelectric stacked actuators using topology optimization, Journal of Intelligent Material Systems and Structures, vol. 3985, pp. 583-591, 2000

Lee W. H., Kang B. H., Oh Y. S., Stephanou H., Sanderson A.C., Skidmore G., Ellis M. (2003), Micropeg manipulation with a compliant microgripper, Proceedings of IEEE Int. Conf. on Robotics and Automation, pp. 3213-3218, Taipei, Taiwan, September 2003

Lim K. B., Gawronski W. (1993), Actuators and sensor placement for control of exible structures, Control and Dynamics Systems: Advances in Theory and Applications, ed. London, Academic Press, 1993

Lim K. B., Gawronski W. (1996), Balanced control of Flexible structures, ed. London, Springer, 1996. 
Maddisetty H., Frecker M. (2002), Dynamic topology optimization of compliant mechanisms and piezoceramic actuators, ASME Journal of Mechanical Design, vol. 126, pp. 975983, 2002

Martin G. D. (1978), On the control of flexible mechanical systems, PhD Dissertation, Stanford University, USA, 1978

Moore B.C. (1981), Principal component analysis in linear systems: controllability, observability, and model reduction, IEEE Transactions on Automatic Control, vol. 26, 1981

Nelli Silva E.C., Kikuchi N. (1999), Design of piezoelectric transducers using topology optimization, Smart Material and Structures, vol. 8, pp. 350 -365, USA, 1999 


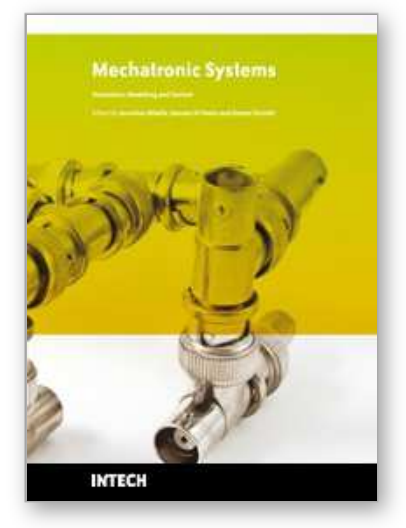

\author{
Mechatronic Systems Simulation Modeling and Control \\ Edited by Annalisa Milella Donato Di Paola and Grazia Cicirelli
}

ISBN 978-953-307-041-4

Hard cover, 298 pages

Publisher InTech

Published online 01, March, 2010

Published in print edition March, 2010

This book collects fifteen relevant papers in the field of mechatronic systems. Mechatronics, the synergistic blend of mechanics, electronics, and computer science, integrates the best design practices with the most advanced technologies to realize high-quality products, guaranteeing at the same time a substantial reduction in development time and cost. Topics covered in this book include simulation, modelling and control of electromechanical machines, machine components, and mechatronic vehicles. New software tools, integrated development environments, and systematic design methods are also introduced. The editors are extremely grateful to all the authors for their valuable contributions. The book begins with eight chapters related to modelling and control of electromechanical machines and machine components. Chapter 9 presents a nonlinear model for the control of a three-DOF helicopter. A helicopter model and a control method of the model are also presented and validated experimentally in Chapter 10. Chapter 11 introduces a planar laboratory testbed for the simulation of autonomous proximity manoeuvres of a uniquely control actuator configured spacecraft. Integrated methods of simulation and Real-Time control aiming at improving the efficiency of an iterative design process of control systems are presented in Chapter 12. Reliability analysis methods for an embedded Open Source Software (OSS) are discussed in Chapter 13. A new specification technique for the conceptual design of self-optimizing mechatronic systems is presented in Chapter 14 . Chapter 15 provides a general overview of design specificities including mechanical and control considerations for micro-mechatronic structures. It also presents an example of a new optimal synthesis method to design topology and associated robust control methodologies for monolithic compliant microstructures.

\title{
How to reference
}

In order to correctly reference this scholarly work, feel free to copy and paste the following:

M. Grossard Mathieu and M. Chaillet Nicolas (2010). Contributions to the Multifunctional Integration for Micromechatronic Systems, Mechatronic Systems Simulation Modeling and Control, Annalisa Milella Donato Di Paola and Grazia Cicirelli (Ed.), ISBN: 978-953-307-041-4, InTech, Available from:

http://www.intechopen.com/books/mechatronic-systems-simulation-modeling-and-control/contributions-to-themultifunctional-integration-for-micromechatronic-systems

\section{INTECH}

open science | open minds

InTech Europe
University Campus STeP Ri

\section{InTech China}

Unit 405, Office Block, Hotel Equatorial Shanghai 
Slavka Krautzeka 83/A

51000 Rijeka, Croatia

Phone: +385 (51) 770447

Fax: +385 (51) 686166

www.intechopen.com
No.65, Yan An Road (West), Shanghai, 200040, China 中国上海市延安西路65号上海国际贵都大饭店办公楼405单元 Phone: +86-21-62489820

Fax: $+86-21-62489821$ 
(C) 2010 The Author(s). Licensee IntechOpen. This chapter is distributed under the terms of the Creative Commons Attribution-NonCommercialShareAlike-3.0 License, which permits use, distribution and reproduction for non-commercial purposes, provided the original is properly cited and derivative works building on this content are distributed under the same license. 